\title{
Application of reduced-port laparoscopic total gastrectomy in gastric cancer preserving the pancreas and spleen
}

\author{
Chikara Kunisaki $\cdot$ Hirochika Makino $\cdot$ Jun Kimura • \\ Ryo Takagawa - Mitsuyoshi Ota - Takashi Kosaka • \\ Hirotoshi Akiyama • Itaru Endo
}

Received: 25 June 2014/ Accepted: 28 October 2014/Published online: 15 November 2014

(C) The International Gastric Cancer Association and The Japanese Gastric Cancer Association 2014

\begin{abstract}
Background The feasibility of using reduced-port laparoscopic total gastrectomy (RPLTG) for the treatment of gastric cancer remains unclear. This study aimed to address the potentially important advantages of this surgical technique.

Methods Between April 2002 and February 2014, 90 patients underwent laparoscopy-assisted total gastrectomies, performed by a single surgeon. Of these, 45 patients underwent RPLTG and 45 patients underwent conventional laparoscopy-assisted total gastrectomy (CLATG). Shortterm outcomes were compared to evaluate the feasibility of RPLTG for gastric cancer.

Results There were several significant differences between the RPLTG and CLATG groups in short-term outcomes: the mean total operation durations were significantly longer in the RPLTG group (319.0 $\mathrm{min}$ ) than in the CLATG group (259.0 min). However, the mean volume of blood loss, the degree of lymph node dissection, and the number of dissected lymph nodes did not differ between the two groups.

Conclusions We have shown that RPLTG could be an acceptable and satisfactory procedure for the treatment of gastric cancer requiring total gastrectomy for surgeons sufficiently experienced in CLATG.
\end{abstract}

C. Kunisaki ( $)$ · H. Makino · J. Kimura - R. Takagawa ·

M. Ota

Department of Surgery, Gastroenterological Center,

Yokohama City University, 4-57 Urafune-cho, Minami-ku,

Yokohama 232-0024, Japan

e-mail: s0714@med.yokohama-cu.ac.jp

T. Kosaka · H. Akiyama · I. Endo

Department of Gastroenterological Surgery, Yokohama City

University, Yokohama, Japan
Keywords Gastric cancer - Laparoscopic total gastrectomy $\cdot$ Reduced-port gastrectomy

\section{Introduction}

Laparoscopy-assisted distal gastrectomy (LADG) for the treatment of gastric cancer has been widely accepted and is widely used in Japan and Korea. Previous studies have reported satisfactory short-term and long-term outcomes for LADG in patients with early gastric cancer [1-5]. Moreover, some recent studies have shown the feasibility of using LADG in advanced gastric cancer patients [6-8]. However, laparoscopy-assisted total gastrectomy (LATG) has not been widely used for the treatment of gastric cancer because of its technical difficulty and complexity, including the reconstruction required and its oncological safety. The most important issue that needs to be resolved is the technique used for the reconstruction of an esophagojejunostomy during LATG $[9,10]$.

Several studies of LATG in gastric cancer patients from large surgical oncology centers have reported favorable short-term and long-term outcomes [11-13], demonstrating that experienced laparoscopic surgeons can perform lymph node dissection and reconstruction as effectively during LATG as during LADG. We expect LATG to become the prevalent technique as surgical skills increase in the foreseeable future.

Recently, surgical outcomes for reduced-port laparoscopic gastrectomy have been reported by several institutions. We showed similar surgical outcomes in a previous study for a reduced-port laparoscopic distal gastrectomy (RPLDG) procedure, using a multiaccess port at the umbilicus plus one additional port, compared with conventional LADG (CLADG), using five ports plus a small, 
5-cm skin incision [14]. Patients who underwent RPLDG were particularly satisfied with the cosmetic appearance of the surgical wounds. Therefore, assessing the feasibility of using reduced-port laparoscopic total gastrectomy (RPLTG) in gastric cancer patients is a rational next step for those who have the skills and willingness to exploit further the potential of this approach. In this study, we have therefore retrospectively compared the short-term outcomes for conventional LATG (CLATG; five ports with a 5-cm skin incision) and RPLTG (a multiaccess port at the umbilicus plus one other port).

\section{Patients and methods}

\section{Patients}

Between April 2002 and February 2014, a total of 645 patients with gastric cancer underwent laparoscopy-assisted gastrectomy with regional lymph node dissection at the Department of Surgery, Gastroenterological Center, Yokohama City University, Japan. Between June 2009 and February 2014, 90 of these patients underwent pancreasand spleen-preserving LATGs, performed by the same surgeon who had previously performed 207 laparoscopic distal gastrectomies, including 34 RPLDGs, 26 laparoscopy-assisted proximal gastrectomies, and 93 CLATGs, before performing the first RPLTG in June 2012. Preoperative diagnosis had established that these were all cases of early gastric cancer. Half of these patients underwent RPLTG and half underwent CLATG and, as this was a retrospective, historical study, it was the former 44 patients who underwent CLATG. Of the latter 46 patients, 45 patients underwent RPLTG and one patient selected CLATG after sufficient explanation of the advantages and disadvantages of CLATG and RPLTG. Surgery was performed only after all possible alternative procedures or treatments had been explained to the patient and informed consent had been obtained. Data for these patients were collected by reviewing their medical records.

The 90 consecutive patients (59 men and 31 women) in this study were aged 35.6-85.4 years (mean 67.6 years), and in all of them gastric adenocarcinoma had been diagnosed before surgery with the use of imaging techniques, after the analysis of endoscopic biopsy specimens. All patients had also undergone a barium swallow test and computed tomography scans. Ultrasonography of the abdomen had been performed in 42 patients $(46.7 \%)$.

The staging and definition of lymph nodes were principally based on the Japanese Classification of Gastric Carcinoma [15] and the lymph node dissection protocol was based on the Japanese Gastric Cancer Guidelines [16]. Splenectomy was performed in patients with early gastric cancer, diagnosed preoperatively as stages I and II. Experienced pathologists in our institution provided high-quality pathological diagnosis service records. Our institutional review board had approved both the use of laparoscopic gastrectomy for tretament of gastric cancer and the retrospective review of patients' records. A range of surgical parameters (such as operation time, volume of blood loss, and number of dissected lymph nodes) and postoperative parameters (such as incidence of morbidity and length of hospital stay) were evaluated.

\section{Surgical procedures}

CLATG with regional lymph node dissection was performed as previously reported [12]. Reconstruction after laparoscopic total gastrectomy used a Roux-en-Y anastomosis, using a transorally inserted anastomotic anvil, OrVil $^{\mathrm{TM}}$ (Covidien), in 45 patients in the CLATG group and 14 patients in the RPLTG group. In the remaining 31 patients in the RPLTG group, the Roux-en-Y anastomosis was formed by hand-sewing a purse-string suture at the esophageal stump followed by the intracorporeal insertion of the anvil head into the esophagus.

In the RPLATG group, a small 40-mm skin incision was made at the umbilicus to insert the OCTO $^{\mathrm{TM}}$ Port V2 (DalimSurgNet, Seoul, Korea), and a $12-\mathrm{mm}$ port was inserted into the right lateral abdomen to support laparoscopic manipulation. The operator stood mainly on the right side of the patient. The OCTO ${ }^{\mathrm{TM}}$ port had four ports, allowing the simultaneous insertion of a $10-\mathrm{mm}$ flexible simple endoscope, an energy device such as a LigaSure ${ }^{\mathrm{TM}}$, and a Roticulator ${ }^{\mathrm{TM}}$ Endograsp $^{\mathrm{TM}}$ II (Covidien). In addition, a DST Series ${ }^{\mathrm{TM}}$ EEA $^{\mathrm{TM}}$ shaft could be introduced through the upper port with a detachable blue cap into the abdominal cavity at the esophagojejunostomy. This port had three functions: a 5-mm port, a 12-mm port, and a 21-28-mm port. The endoscope was routinely inserted through the lower portion of the square, the energy device through the upper portion, and the endograsp through the right side of the port (Fig. 1). Throughout laparoscopic manipulations, the energy device was usually held in the right hand. However, sometimes it was held in the left hand and inserted through the $12-\mathrm{mm}$ port in the right lateral abdomen, when it was necessary to dissect tissue in a tangential direction and subsequently necessary to avoid injuring organs. For example, suprapancreatic lymph nodes could be most easily dissected using left-hand manipulations. In addition, sometimes when the energy device was held in the right hand and the forceps in the left hand, the operator was forced to cross the instruments and this could interfere with the surgeon's manipulations. In such situations, changing the hand holding the energy device allowed us to perform the procedure comfortably. 


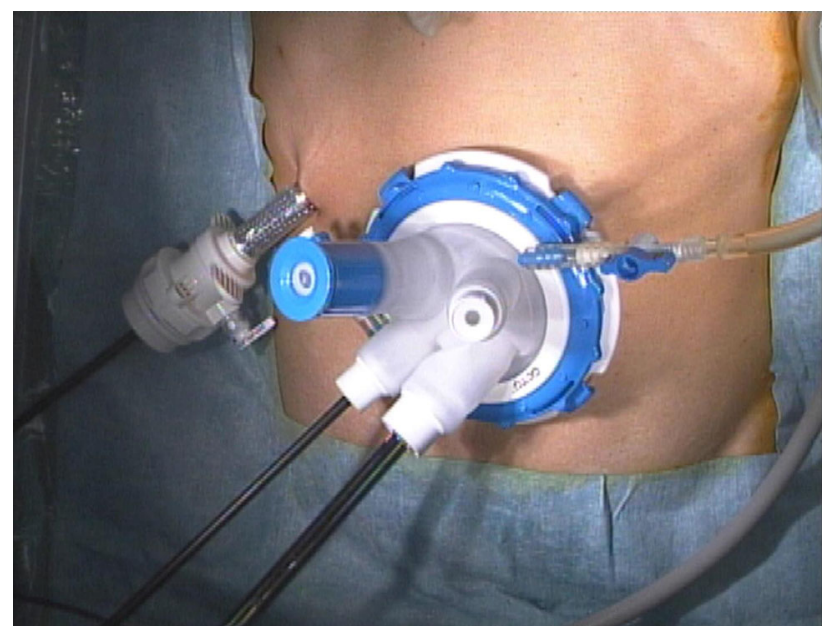

Fig. 1 Photograph showing the position of the ports and devices used for reduced-port laparoscopic total gastrectomy. The OCTO ${ }^{\mathrm{TM}}$ port was inserted in a 4-cm incision at the umbilicus and had 5-, 12-, and 21-28-mm ports. An additional $12-\mathrm{mm}$ port was inserted in the right lateral abdomen

In CLATG, the so-called triangle formation achieved by retracting the tissues with three forceps with appropriate strengths has been considered essential for safe and effective dissection. However, in RPLTG, a surgical team is equipped with a maximum of two forceps to retract tissues. Thus, it is important to use the Roticulator ${ }^{\mathrm{TM}}$ grasper to retract tissue in the optimal direction with sufficient force, and sometimes to make use of tissue adhesion or gravity.

After lymph node dissection, the stomach was resected within the abdomen using an Endo GIA ${ }^{\mathrm{TM}}$ Ultra universal stapler, a straight cut at the junction with the abdominal esophagus, and extraction through the umbilical incision. After a purse-string suture had been had sewn at the abdominal esophageal stump within the abdomen, an anvil head was inserted intracorporeally and securely attached. A jejunal loop was made extracorporeally through the $\mathrm{OCTO}^{\mathrm{TM}}$ port and brought up behind the colon and through the transverse mesocolon intracorporeally. A DST Series ${ }^{\mathrm{TM}} \mathrm{EEA}^{\mathrm{TM}}$ shaft was inserted into the jejunal loop extracorporeally, and the jejunum was secured to the shaft with a rubber band. A DST Series ${ }^{\mathrm{TM}}$ EEA $^{\mathrm{TM}}$ shaft was introduced into the abdominal cavity through the upper side port of the $\mathrm{OCTO}^{\mathrm{TM}}$ port to maintain a pneumoperitoneum. The esophagojejunostomy could be completed without making the small skin incision at the upper abdomen used in the CLATG group. The jejunal stump was sutured intracorporeally using an Endo GIA ${ }^{\mathrm{TM}}$ universal stapler (60-2.5). To create a 40 -m Roux-en-Y limb, the transected jejunum was used extracorporeally to form a side-to-side jejunojejunostomy using a DSTSeries $^{\mathrm{TM}} \mathrm{EEA}^{\mathrm{TM}}$ stapler (25-3.5; Covidien). The jejunal

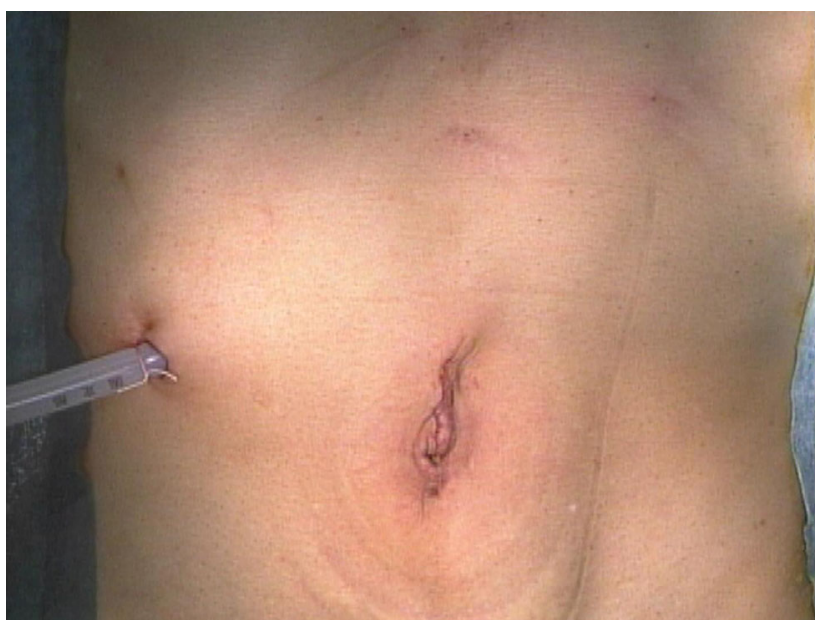

Fig. 2 Photograph showing a patient's abdomen on completion of reduced-port laparoscopic total gastrectomy. A silicon drain was inserted at the site of the port in the right lateral abdomen and the 4-cm skin incision was closed meticulously

stump was also sutured extracorporeally using an Endo GIA $^{\mathrm{TM}}$ universal stapler (60-2.5). After the abdomen had been irrigated with $3,000 \mathrm{~mL}$ irrigation fluid, one silicon drain was placed behind the esophagojejunal anastomosis (Fig. 2).

\section{Postoperative care}

All patients in both groups received epidural analgesia for 3 days after the operation. However, if a patient required additional pain control during or after administration of the epidural, nonsteroidal anti-inflammatory drugs were administered. Before March 2010, patients resumed an oral diet on day 6 after surgery and were discharged on day 14 , in accordance with the clinical pathway used at our institution, provided there was no morbidity and their food intake was sufficient. The clinical pathway then changed and an oral diet commenced on day 4 after surgery,and patients were discharged on day 12 .

After discharge, all 90 patients were asked by the surgeon to grade their satisfaction with the cosmetic appearance of their scars as low, moderate, or high.

\section{Statistical analysis}

Data were analyzed using the SPSS statistical software program (SPSS, Chicago, IL, USA). Patient characteristics were compared using the two-tailed Fisher exact test or the chi square test with the Yates correction. Quantitative variables were compared using Student's $t$ test and were expressed as the mean \pm standard deviation. Probability ( $p$ ) values of less than 0.05 were considered to be statistically significant. 
Table 1 Comparison of the characteristics of patients in the reduced-port laparoscopic total gastrectomy (RPLTG) and conventional laparoscopyassociated total gastrectomy (CLATG) groups
Ranges are given in parentheses

ASA American Society of Anesthesiologists

${ }^{a}$ Slightly depressed, elevated type, and elevated plus depressed type

\begin{tabular}{|c|c|c|c|}
\hline & RPLTG $(n=45)$ & CLATG $(n=45)$ & $p$ value \\
\hline Age (years) & $67.1 \pm 10.9$ & $67.4 \pm 10.9$ & 0.911 \\
\hline Median (range) & $68.4(36-84)$ & $67.0(36-85)$ & \\
\hline Gender (male/female) & $28 / 17$ & $31 / 14$ & 0.658 \\
\hline Body mass index $\left(\mathrm{kg} / \mathrm{m}^{2}\right)$ & $22.7 \pm 3.5$ & $22.3 \pm 2.5$ & 0.582 \\
\hline Median (range) & $23.6(14.7-32.4)$ & $22.9(17.0-27.5)$ & \\
\hline ASA classification & & & 0.666 \\
\hline 1 & 17 & 21 & \\
\hline 2 & 25 & 22 & \\
\hline 3 & 3 & 2 & \\
\hline Prognostic nutritional index & $54.5 \pm 5.2$ & $54.5 \pm 5.5$ & 0.997 \\
\hline \multicolumn{4}{|l|}{ Past history of laparotomy } \\
\hline Present & 7 & 9 & 0.784 \\
\hline Cholecystectomy & 1 & 2 & \\
\hline Appendectomy & 4 & 5 & \\
\hline Gynecological & 4 & 2 & \\
\hline \multicolumn{4}{|l|}{ Comorbid disease } \\
\hline Present & 28 & 24 & 0.522 \\
\hline Cardiovascular & 21 & 16 & \\
\hline Hyperlipidemia & 12 & 6 & \\
\hline Diabetes mellitus & 3 & 2 & \\
\hline Respiratory & 2 & 2 & \\
\hline Cerebrovascular & 2 & 2 & \\
\hline Other cancer & 1 & 6 & \\
\hline Others & 1 & 1 & \\
\hline Location of tumor & & & 0.011 \\
\hline Middle third & 11 & 25 & \\
\hline Upper third & 31 & 18 & \\
\hline Entire stomach & 3 & 2 & \\
\hline Macroscopic appearance & & & 0.393 \\
\hline Superficial $^{\mathrm{a}}$ & 38 & 42 & \\
\hline Well defined & 3 & 1 & \\
\hline Ill defined & 4 & 2 & \\
\hline Tumor diameter $(\mathrm{mm})$ & $42.1 \pm 27.6$ & $36.9 \pm 18.5$ & 0.297 \\
\hline Histological type & & & 0.382 \\
\hline Differentiated & 26 & 31 & \\
\hline Undifferentiated & 19 & 14 & \\
\hline Depth of invasion & & & 0.858 \\
\hline Mucosa, submucosa & 35 & 37 & \\
\hline Proper muscle, subserosa & 9 & 7 & \\
\hline Serosa exposed & 1 & 1 & \\
\hline Lymph node metastasis present & 7 & 7 & 1.000 \\
\hline Stage & & & 0.010 \\
\hline IA & 30 & 35 & \\
\hline IB & 7 & 4 & \\
\hline IIA & 7 & 0 & \\
\hline IIB & 1 & 1 & \\
\hline IIIA & 0 & 5 & \\
\hline
\end{tabular}


Table 2 Comparison of the surgical outcomes of patients in the reduced-port laparoscopic total gastrectomy $(R P L T G)$ and the conventional laparoscopy-associated total gastrectomy $(C L A T G)$ groups

\begin{tabular}{lllr}
\hline & $\begin{array}{l}\text { RPLTG } \\
(n=45)\end{array}$ & $\begin{array}{l}\text { CLATG } \\
(n=45)\end{array}$ & $p$ value \\
\hline $\begin{array}{l}\text { Lymph node dissection } \\
\text { D1 plus no. 8a, 9, 11p }\end{array}$ & 16 & 25 & 0.090 \\
$\begin{array}{l}\text { D1 plus no. 8a, 9, 11p, } \\
\quad 29\end{array}$ & 20 & \\
$\quad$ 11d, 12a & & & \\
$\begin{array}{l}\text { Total operation time (min) } \\
\text { Total blood loss (mL) }\end{array}$ & $319.0 \pm 45.3$ & $259.0 \pm 34.1$ & $<0.001$ \\
$\begin{array}{l}\text { No. of lymph nodes } \\
\text { dissected }\end{array}$ & $46.3 \pm 75.1$ & $118.9 \pm 83.5$ & 0.137 \\
\hline
\end{tabular}

\section{Results}

\section{Patient characteristics}

The characteristics of the 90 patients enrolled in this study are shown in Table 1. There were no significant differences between the CLATG and RPLTG groups in age, sex, body mass index, the American Society of Anesthesiologists physical status classification, the prognostic nutritional index, the presence of comorbidities, or prior abdominal surgery. Pathologically, we found that tumors mainly located in the upper third of the stomach and more advanced tumors were significantly more frequent in the RPLTG group than in the CLATG group. By contrast, the macroscopic appearance, tumor diameter, histological type, depth of invasion, and the extent of lymph node metastasis did not show significant differences between the two groups.

\section{Surgical characteristics}

There were significant differences in the total operation time. However, the total blood loss, the extent of lymph node dissection, and the number of lymph nodes retrieved did not differ between the two groups (Table 2).

\section{Postoperative recovery}

As shown in Table 3, the time to resumption of an oral diet and the length of stay in hospital postoperatively were shorter in the RPLTG group although the clinical pathway was different between the two groups. The peak value of C-reactive protein was significantly higher in the CLATG group owing to the high incidence of anastomotic and duodenal leakage. However, cosmetic satisfaction was significantly greater in the RPLTG group than in the CLATG group $(p=0.021)$. No significant differences were observed between the two groups in the peak value of the white blood cell (WBC) count, the presence of liver dysfunction, the time to first flatus, the time for which nonsteroidal anti-inflammatory drugs were used, and postoperative morbidity. Death was not observed in the two groups.

\section{Discussion}

Reduced-port gastrectomy can sometimes create conflicts between different surgical devices and may make precise manipulations more difficult to perform. Therefore, the application of this technique to such a technically complex surgery as laparoscopic gastrectomy may be considered inappropriate. However, we have shown in this retrospective study that our RPLTG technique for treatment of gastric cancer resulted in acceptable and satisfactory shortterm patient outcomes compared with those achieved using a conventional laparoscopic technique.

The application of CLATG for treatment of gastric cancer has also been reported [11-13]. The surgical procedure for CLATG is more complex and challenging than that for laparoscopic distal gastrectomy, even for an experienced gastric surgeon. In particular, the reconstruction technique has not yet been well established in CLATG $[9,10]$.

In all laparoscopic surgery, adequate retraction and counter-retraction are essential to control the orientation of organs during surgery and prevent intraoperative complications. In reduced-port gastrectomy, it is sometimes difficult to manipulate organs in the appropriate direction with appropriate force. It is therefore essential that surgeons have sufficient education, training, and experience in performing gastrectomies, including laparoscopic procedures, to be able to perform complex reduced-port gastrectomy safely. Optimal organ retraction provides surgeons with good visibility within the abdomen and enables them to dissect lymph nodes safely and create intracorporeal anastomoses within an acceptable time and with acceptable blood loss, and so subsequently reduces the incidence of postoperative complications. In CLATG, adequate retraction in the required direction can be performed by an assistant surgeon using two forceps in addition to the single forceps manipulated by the operator. In RPLTG, the Roticulator $^{\mathrm{TM}}$ grasper used throughout the operation by the assistant surgeon and the forceps used by the operator, and using adhesion between tissues or organs, provide us with an acceptable intra-abdominal view. The organs and tissues were retracted ventrally using a Roticulator ${ }^{\mathrm{TM}}$ grasper, and this manipulation allowed meticulous dissection of lymph nodes and tissues, by avoiding conflicts between different devices. It was also important to change the hand holding the energy device if it was thought necessary and useful. 
Table 3 Comparison of the postoperative recovery of patients in the reduced-port laparoscopic total gastrectomy (RPLTG) and the conventional laparoscopy-associated total gastrectomy (CLATG) groups

Ranges are given in parentheses

$A L T$ alanine aminotransferase, AST aspartate aminotransferase, NSAIDs nonsteroidal antiinflammatory drugs, $W B C$ white blood cell

\begin{tabular}{|c|c|c|c|}
\hline & RPLTG $(n=45)$ & CLATG $(n=45)$ & $p$ value \\
\hline Peak C-reactive protein (mg/dL) & $11.9 \pm 5.6$ & $16.5 \pm 6.8$ & 0.001 \\
\hline Peak WBC $\left(/ \mathrm{m}^{3}\right)$ & $12,385 \pm 4,080$ & $12,760 \pm 4,530$ & 0.682 \\
\hline Peak ALT (U/L) & $120.9 \pm 115.7$ & $144.2 \pm 201.9$ & 0.505 \\
\hline Peak AST (U/L) & $122.2 \pm 106.9$ & $161.6 \pm 170.7$ & 0.366 \\
\hline Time to first flatus (days) & $2.6 \pm 1.9$ & $2.9 \pm 1.2$ & 0.432 \\
\hline Median time to first flatus (days) & $3.0(2-5)$ & $3.0(2-4)$ & \\
\hline No. of doses of NSAIDs used & $5.7 \pm 2.0$ & $6.0 \pm 5.3$ & 0.258 \\
\hline Median no. of doses of NSAIDs used & $5.0(2-7)$ & $6.0(4-8)$ & \\
\hline Time to resumption of oral diet (days) & $4.8 \pm 2.4$ & $7.5 \pm 4.9$ & 0.001 \\
\hline Median (range) & $4(4-16)$ & $5(4-29)$ & \\
\hline Morbidity & 6 & 13 & 0.120 \\
\hline Anastomotic leakage & 2 & 5 & \\
\hline Duodenal stump leakage & 1 & 2 & \\
\hline Surgical site infection & 2 & 2 & \\
\hline Pneumonia & 0 & 3 & \\
\hline Cholecystitis & 0 & 1 & \\
\hline Anastomotic stenosis & 1 & 1 & \\
\hline Mortality & 0 & 0 & 1.000 \\
\hline Postoperative hospital stay (days) & $16.3 \pm 8.9$ & $27.0 \pm 19.1$ & 0.008 \\
\hline Median postoperative hospital stay (days) & $13.0(11-41)$ & $19.0(12-114)$ & \\
\hline Cosmetic satisfaction & & & 0.021 \\
\hline Low & 0 & 3 & \\
\hline Moderate & 7 & 15 & \\
\hline High & 38 & 27 & \\
\hline
\end{tabular}

When dissecting the infrapyloric lymph nodes, we usually revealed and dissected the right gastroepiploic vein using the right hand, and similarly dissected the lymph nodes around the right gastroepiploic artery using the left hand, to prevent injury to the pancreas. This procedure is difficult and sometimes lengthened the operation. When dissecting lymph nodes along the suprapancreatic arteries, we found that using the left hand was safe and smooth while retracting the pancreas to the dorsal side using the right hand.

Successful results using single-port or reduced-port laparoscopic surgery for distal gastrectomies in patients with early gastric cancer have been reported previously [17-19]. An experienced and skillful surgeon can perform such complex laparoscopic gastrectomies after gaining sufficient experience of conventional laparoscopic gastrectomies. In our previous study [14], we also reported satisfactory outcomes for reduced-port (SILS Port ${ }^{\mathrm{TM}}$ plus one port) LADG.

In this study, we found that the technical difficulty of this operation resulted in a longer overall operation time, with more time spent on lymph node dissection, compared with CLATG although the time taken for reconstruction did not differ significantly between the two groups. We used an $\mathrm{OCTO}^{\mathrm{TM}}$ port and added a $12-\mathrm{mm}$ port in the lateral abdomen, which could be used as a drain after surgery. The addition of this one extra port enabled us to perform lymph node dissection laparoscopically to a satisfactory and sufficient degree, although this took longer to complete than in CLATGs. It may be difficult to shorten the operation time for RPLTG even for an experienced laparoscopic surgeon.

During this study, we encountered seven cases of anastomotic leakage after using the $\mathrm{OrVil}^{\mathrm{TM}}$ device and we therefore changed the method used to a hand-sewn, pursestring suture technique. Since then, we have had no further cases of leakage. However, there is an urgent need to develop a reliable technique using an innovative device for performing esophagojejunostomy.

Both the time to the resumption of an oral diet and the length of stay in hospital postoperatively were significantly longer in the CLATG groups. Postoperative morbidity and our institution's current clinical pathway affected these results, and so it may be difficult to emphasize the advantage of RPLTG.

The observation that the number of lymph nodes dissected was the same in both patient groups suggested that lymph node dissection in the RPLTG group was done to an 
acceptable standard. This also suggested that the long-term survival for these patients may be expected to be equivalent in the two groups, although follow-up studies are needed to confirm this.

Some previous studies have shown that totally laparoscopic total gastrectomy (TLTG) using either functional end-to-end esophagojejunostommy or $\mathrm{OrVil}^{\mathrm{TM}}$ reconstructions is an acceptable surgical procedure [20, 21]. Blood losses and postoperative morbidities in these studies were similar to those in our study, although our operation times were longer than those in the TLTG studies. Although RPLTG does not need additional three-port sites compared with the TLTG procedure, this technique does need great laparoscopic skills and longer operation times. The fundamental difference between TLTG and RPLTG is the number of very small incisions for ports although the resected specimen is similarly retrieved through the umbilical wound in both methods. By contrast, satisfaction of patients in the RPLTG group may depend on the small umbilical wound instead of the minilaparotomy in the upper abdomen in the CLATG group rather than the number of ports. Therefore, this study looked at a mixture of the CLATG versus TLTG comparison and CLATG versus RPLTG comparison. This is a clear weakness and limitation of this retrospective study. In our institution, we initially made the Roux-en- $\mathrm{Y}$ anastomosis through a $10-\mathrm{cm}$ skin incision made after LATG. We then used an $\mathrm{OrVil}^{\mathrm{TM}}$ device for the esophagojejunostomy through a small, 4-5$\mathrm{cm}$ skin incision, and more recently we have used an Or$\mathrm{Vil}^{\mathrm{TM}}$ device for an intracorporeal esophagojejunostomy using the reduced-port technique. Most recently, we have inserted an anvil head after creating a hand-sewn, pursestring suture of the esophageal stump, which was secured intracorporeally, and subsequently we were able to complete the esophagojejunostomy as quickly as an open esophagojejunostomy. Introducing these complex surgical procedures step by step would be ideal to achieve a steady and safe surgical learning curve. However, it is difficult to conclude that RPLTG reduced the incidence of anastomotic leakage and subsequently shortened the hospital stay because the anastomotic methods were changed with time in this retrospective study.

As we have shown in this study, cosmetic satisfaction was obtained in a subset of patients receiving RPLTG. Therefore, it is important to explain the advantages and disadvantages of this technique to patients before surgery.

\section{Conclusion}

Our results suggest that RPLTG for treatment of gastric cancer may be an acceptable and satisfactory technique although this retrospective study has some limitations. This method may be one of the operative methods for treatment of gastric cancer requiring or indicating laparoscopic total gastrectomy by some experienced laparoscopic surgeons.

\section{References}

1. Lee SI, Choi YS, Park DJ, Kim HH, Yang HK, Kim MC. Comparative study of laparoscopy-assisted distal gastrectomy and open distal gastrectomy. J Am Coll Surg. 2006;202:874-80.

2. Viñuela EF, Gonen M, Brennan MF, Coit DG, Strong VE. Laparoscopic versus open distal gastrectomy for gastric cancer: a meta-analysis of randomized controlled trials and high-quality nonrandomized studies. Ann Surg. 2012;255:446-56.

3. Takiguchi S, Fujiwara Y, Yamasaki M, Miyata H, Nakajima K, Sekimoto M, et al. Laparoscopy-assisted distal gastrectomy versus open distal gastrectomy. A prospective randomized singleblind study. World J Surg. 2013;37:2379-86.

4. Kunisaki C, Makino H, Kosaka T, Oshima T, Fujii S, Takagawa $\mathrm{R}$, et al. Surgical outcomes of laparoscopy-assisted gastrectomy versus open gastrectomy for gastric cancer: a case-control study. Surg Endosc. 2012;26:804-10.

5. Fukunaga T, Hiki N, Kubota T, Nunobe S, Tokunaga M, Nohara $\mathrm{K}$, et al. Oncologic outcomes of laparoscopy-assisted distal gastrectomy for gastric cancer. Ann Surg Oncol. 2013;20:2676-82.

6. Zhao Y, Yu P, Hao Y, Qian F, Tang B, Shi Y, et al. Comparison of outcomes for laparoscopically assisted and open radical distal gastrectomy with lymphadenectomy for advanced gastric cancer. Surg Endosc. 2011;25:2960-6.

7. Huang JL, Wei HB, Zheng ZH, Wei B, Chen TF, Huang Y, et al. Laparoscopy-assisted D2 radical distal gastrectomy for advanced gastric cancer. Dig Surg 2010;27:291-6.

8. Park do J, Han SU, Hyung WJ, Kim MC, Kim W, Ryu SY, et al. Korean Laparoscopic Gastrointestinal Surgery Study (KLASS) Group. Long-term outcomes after laparoscopy-assisted gastrectomy for advanced gastric cancer: a large-scale multicenter retrospective study. Surg Endosc. 2012;26:1548-53.

9. Lee SW, Nomura E, Bouras G, Tokuhara T, Tsunemi S, Tanigawa N. Long-term oncologic outcomes from laparoscopic gastrectomy for gastric cancer: a single-center experience of 601 consecutive resections. J Am Coll Surg. 2010;211:33-40.

10. Jeong O, Ryu SY, Zhao XF, Jung MR, Kim KY, Park YK. Short term surgical outcomes and operative risks of laparoscopic total gastrectomy (LTG) for gastric carcinoma: experience at a large volume center. Surg Endosc. 2012;26:3418-25.

11. Kim KH, Kim YM, Kim MC, Jung GJ. Is laparoscopy-assisted total gastrectomy feasible for the treatment of gastric cancer? A case-matched study. Dig Surg. 2013;30:348-54.

12. Kunisaki C, Makino H, Oshima T, Fujii S, Kimura J, Takagawa R, et al. Application of the transorally inserted anvil (OrVil) after laparoscopy-assisted total gastrectomy. Surg Endosc. 2011;25:1300-5.

13. LaFemina J, Viñuela EF, Schattner MA, Gerdes H, Strong VE. Esophagojejunal reconstruction after total gastrectomy for gastric cancer using a transorally inserted anvil delivery system. Ann Surg Oncol. 2013;20:2975-83.

14. Kunisaki C, Ono HA, Oshima T, Makino H, Akiyama H, Endo I. Relevance of reduced-port laparoscopic distal gastrectomy for gastric cancer: a pilot study. Dig Surg. 2012;29:261-8.

15. Japanese Gastric Cancer Association. Japanese classification of gastric carcinoma: 3rd English edition. Gastric Cancer. 2011;14: 101-12.

16. Association Japanese Gastric Cancer. Japanese gastric cancer treatment guidelines 2010 (ver. 3). Gastric Cancer. 2011;14: 113-23. 
17. Omori T, Oyama T, Akamatsu H, Tori M, Ueshima S, Nishida T. Transumbilical single-incision laparoscopic distal gastrectomy for early gastric cancer. Surg Endosc. 2011;25:2400-4.

18. Kawamura H, Tanioka T, Shibuya K, Tahara M, Takahashi M. Comparison of the invasiveness between reduced-port laparoscopy-assisted distal gastrectomy and conventional laparoscopyassisted distal gastrectomy. Int Surg. 2013;98:247-53.

19. Kawamura H, Tanioka T, Kuji M, Tahara M, Takahashi M. The initial experience of dual port laparoscopy-assisted total gastrectomy as a reduced port surgery for total gastrectomy. Gastric Cancer. 2013;16:602-8.
20. Ebihara Y, Okushiba S, Kawarada Y, Kitashiro S, Katoh H. Outcome of functional end-to-end esophagojejunostomy in totally laparoscopic total gastrectomy. Langenbecks Arch Surg. 2013;398:475-9.

21. Ito $\mathrm{H}$, Inoue $\mathrm{H}$, Odaka $\mathrm{N}$, Satodate $\mathrm{H}$, Onimaru $\mathrm{M}$, Ikeda $\mathrm{H}$, et al. Evaluation of the safety and efficacy of esophagojejunostomy after totally laparoscopic total gastrectomy using a trans-orally inserted anvil: a single-center comparative study. Surg Endosc. 2014. doi:10.1007/s00464-014-3417-x. 